\title{
Correction to: contrasting effects of urbanization on arboreal and ground-dwelling land snails: role of trophic interactions and habitat fragmentation
}

\author{
Ikuyo Saeki ${ }^{1}$ (D) Shigeru Niwa ${ }^{2} \cdot$ Noriyuki Osada $^{3} \cdot$ Wakana Azuma $^{4} \cdot$ Tsutom Hiura $^{5}$
}

Published online: 3 March 2020

(C) Springer Science+Business Media, LLC, part of Springer Nature 2020

\section{Correction to: Urban Ecosyst} https://doi.org/10.1007/s11252-020-00930-6

The original version of the article unfortunately contained mistakes in the legends of Fig. 3.

The legends (1) Blue square: Euhadra brandtii sapporo, ground is changed to Blue square: Euhadra brandtii sapporo, canopy (2) Red circle: Euhadra brandtii sapporo, canopy is changed to Red circle: Euhadra brandtii sapporo, ground.

The authors apologize for this mistake and the updated figure is shown below.

The online version of the original article can be found at https://doi.org/ 10.1007/s11252-020-00930-6

\footnotetext{
Ikuyo Saeki

saeki.ikuyo.ge@u.tsukuba.ac.jp

1 Faculty of Life and Environmental Sciences, University of Tsukuba, 1-1-1 Tennodai, Tsukuba, Ibaraki 305-8571, Japan

2 Japan Wildlife Research Center, 3-3-7 Kotobashi, Sumida-ku, Tokyo 130-8606, Japan

3 Laboratory of Plant Conservation Science, Faculty of Agriculture, Meijo University, Nagoya, Aichi 468-8502, Japan

4 Present address: Graduate School of Agricultural Science, Kobe University, Kobe, Hyogo 657-8501, Japan

5 Field Science Center, Hokkaido University, Kita 9 Nishi 9, Sapporo, Hokkaido 060-0809, Japan
} 
Fig. 3 Comparison of survival curves of Euhadra brandtii sapporo and Ezohelix gainesi for canopy and ground treatments in the 2017-fall (left) and 2018-

summer (right) experiments.

Error bars indicate $1 \mathrm{SE}$ based on results of 8 samples $\times 3$ sites (i.e., $n=24$ ) for one forest type. See Table 2 for results of pairwise comparisons by log-rank tests

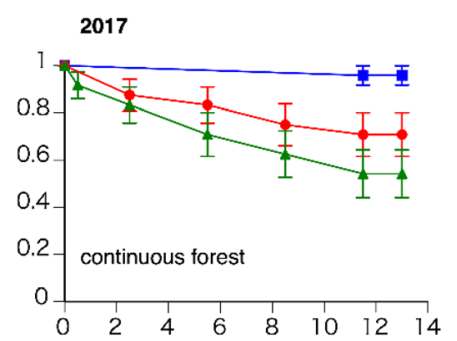

2018
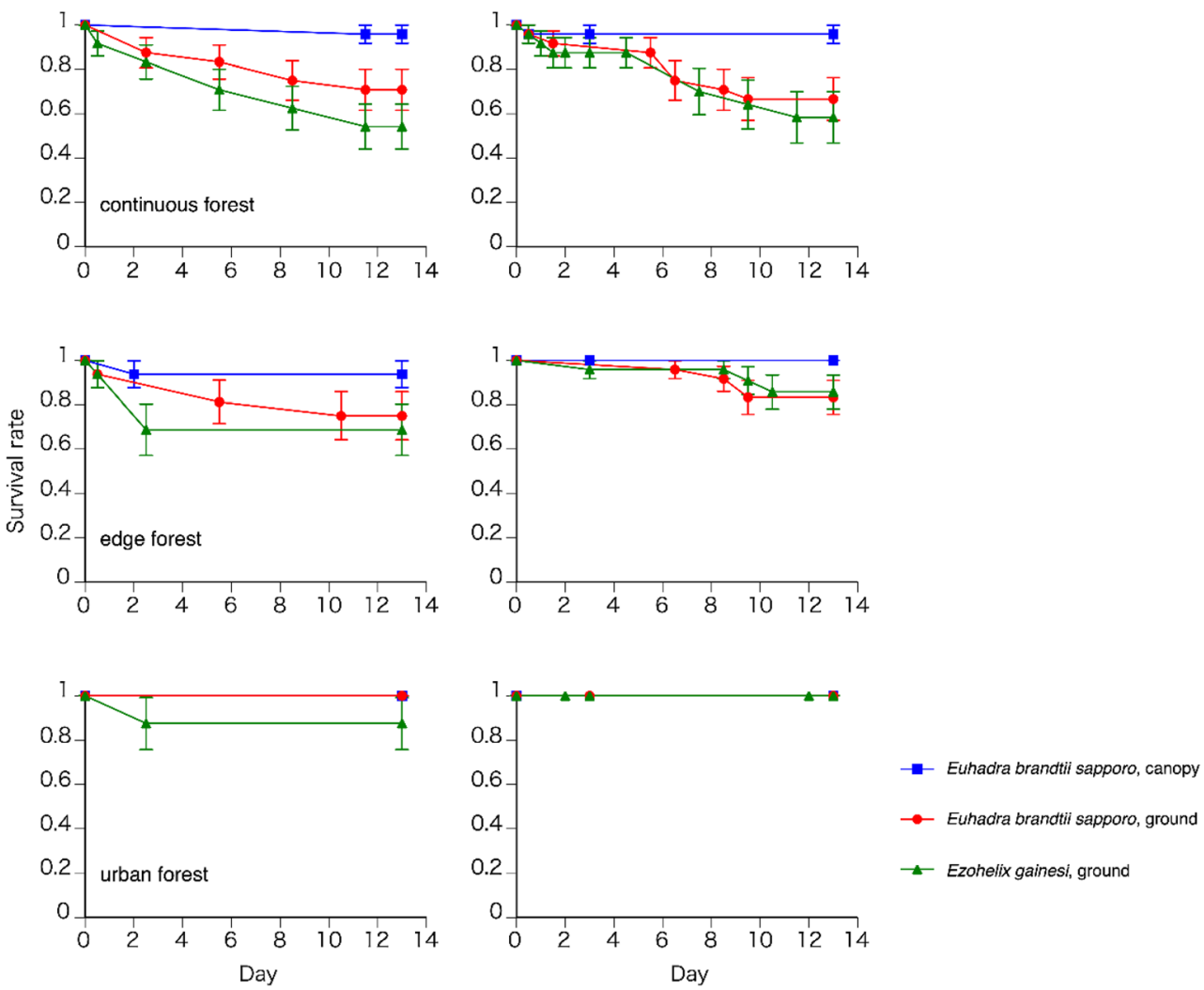